\title{
Induction of Th1/Th2-Balanced Protection Against SARS-CoV-2 Through Mucosal Delivery of An Adenovirus Vaccine Expressing an Engineered Spike Protein
}

\author{
Nai-Hsiang Chung \\ National Health Research Institutes \\ Ying-Chin Chen \\ National Health Research Institutes \\ Shiu-Ju Yang \\ National Health Research Institutes \\ Yu-Ching Lin \\ National Health Research Institutes \\ Horng-Yunn Dou \\ National Health Research Institutes \\ Ching-Len Liao \\ National Health Research Institutes \\ Yen-Hung Chow ( $\nabla$ choeyenh@nhri.edu.tw) \\ National Health Research Institutes
}

\section{Research Article}

Keywords: SARS-CoV-2, immunogenicity, adenovirus, vaccine

Posted Date: January 15th, 2021

DOl: https://doi.org/10.21203/rs.3.rs-134494/v1

License: (c) (i) This work is licensed under a Creative Commons Attribution 4.0 International License. Read Full License

Version of Record: A version of this preprint was published at Vaccine on December 1st, 2021. See the published version at https://doi.org/10.1016/j.vaccine.2021.12.024. 
Induction of Th1/Th2-balanced protection against SARS-CoV-2 through mucosal delivery of an adenovirus vaccine expressing an engineered spike protein

Nai-Hsiang Chung ${ }^{1,2}$, Ying-Chin Chen ${ }^{1}$, Shiu-Ju Yang ${ }^{1}$, Yu-Ching Lin $^{1}$, Horng-Yunn Dou ${ }^{1}$, Ching-Len Liao ${ }^{1}$, Yen-Hung Chow ${ }^{1,3^{*}}$

${ }^{1}$ National Institute of Infectious Disease and Vaccinology, National Health Research Institutes, Zhunan, Taiwan; ${ }^{2}$ Graduate Program of Biotechnology in Medicine, Institute of Molecular and Cellular Biology, National Tsing Hua University, Hsinchu, Taiwan; ${ }^{3}$ Graduate Institute of Biomedical Sciences, China Medical University, Taichung, Taiwan.

Key words:

SARS-CoV-2; immunogenicity; adenovirus; vaccine

\section{Running title:}

Different immunization routes influence the efficacy of the AdCoV-2 vaccine against SARS-CoV-2

\section{Corresponding Author:}

Yen-Hung Chow

Room No. R1-7033, No. 35, Keyan Road, Zhunan Town, Miaoli County 350, Institute of Infectious Disease and Vaccinology, National Health Research Institutes, Taiwan Tel: 886-37-246166 ext. 37738

Email: choeyenh@nhri.edu.tw 


\section{Abstract}

We developed a series of recombinant human type 5 adenoviruses that express the full-length or membrane-truncated spike protein (S) of SARS-CoV-2 (AdCoV2-S or AdCoV2-SdTM, respectively). We tested the immunoprotective efficacy against SARS-CoV-2 via intranasal (i.n.) or subcutaneous (s.c.) immunization in a rodent model following two-dose immunizations. Mucosal delivery of adenovirus (Ad) vaccines could induce anti-SARS-CoV-2 IgG and IgA in the serum and in the mucosal, respectively as indicated by vaginal wash (vw). Serum anti-SARS-CoV-2 IgG but not IgA was induced in the vw by s.c. injection of AdCoV2-S. Intranasal administration of AdCoV2-S was able to induce higher anti-SARS-CoV-2 antibody levels than s.c. injection. Immunization with AdCoV2-SdTM induced a lower antibody response than AdCoV2-S. In addition, the degree of neutralization of clinically isolated SARS-CoV-2 in the serum correlated with the above anti-SARS-CoV-2 responses; the most potent neutralizing activity was observed in the AdCoV2-S i.n. group, and less viral neutralizing activity was observed in response to AdCoV2-S s.c. and AdCoV2dTM i.n. Novelty, S-specific IgG1 which represented Th2-mediated humoral response was dominantly induced in Ad i.n.-immunized serum in contrast to more IgG2a which represented Th1-mediated cellular response found in Ad s.c.-immunized serum. The activation of S-specific IFN- $\gamma$ and IL-4 in Th1 and Th2 cells, respectively, was observed in the AdCoV2s i.n. and s.c. groups, indicating the Th1/Th2-balenced immunity was activated. During the protection study, two doses of i.n. AdCoV2-S or i.n. AdCoV2-SdTM significantly prevented body weight loss and reduced pulmonary viral loads in hamsters. A significant reduction in inflammation in the lungs was observed in AdCoV-S-immunized hamsters following a SARS-CoV-2 challenge. It correlated to Th1 cytokine but no inflammatory cytokines secretions found in i.n. AdCoV-immunized respiratory tract. These results indicate that intranasal delivery of 
AdCoV2-S vaccines is safe and potent at preventing SARS-CoV-2 infections. 


\section{Introduction}

COVID-19 is an emerging respiratory infectious disease that caused by severe acute respiratory syndrome coronavirus 2 (SARS-CoV-2). SARS-CoV-2 is efficiently transmitted from person to person and has thus been able to spread rapidly across all continents globally. To date, no effective medications are used clinically; therefore, the development of a safe and effective treatment for COVID-19 is urgently needed.

There are three coronaviruses that cause deadly pneumonia in humans: severe acute respiratory syndrome coronavirus $(\mathrm{SARS}-\mathrm{COV})^{1}$, Middle-East respiratory syndrome coronavirus (MERS-CoV) ${ }^{2}$, and SARS-CoV-2 ${ }^{3}$. SARS-CoV, MERS-CoV, and SARS-CoV- 2 all belong to the $\beta$-coronavirus genus. The genome of SARS-CoV-2 is 96.2\% identical to that of bat CoV RaTG13, whereas it is $79.5 \%$ identical to that of SARS-CoV ${ }^{4}$. The coronavirus is an enveloped virus containing a positive single-stranded RNA associated with a lipid membrane derived from the host cell. The coronavirus has the largest RNA genome among all the known RNA viruses ${ }^{4}$. Coronavirus encodes the spike (S) protein, which forms homotrimers that protrude from the surface of viral particles and is used for entry into host cells ${ }^{5}$. During viral replication in the infected cell, translated premature $S$ protein is cleaved at the boundary between the $S_{1}$ and $S_{2}$ subunits, which remain noncovalently bound in the prefusion conformation ${ }^{6} . \mathrm{S} 1$ is responsible for binding to the host cell receptor, and $\mathrm{S} 2$ is responsible for the fusion of the viral and cellular membranes after S1-receptor interactions occur ${ }^{6,7}$. Recent studies have indicated that SARS-CoV and several SARS-related coronaviruses, including SARS-CoV-2, interact directly with angiotensin-converting enzyme 2 (ACE2) via S1 to enter target cells ${ }^{8}$.

A replication-incompetent adenoviral vector (Ad) with a recombinant E1-deficient Ad carrying a transgene has been shown to be a potential vaccine vector in multiple successful preclinical and clinical studies ${ }^{9-11}$. Ad is a strong DC activator 
that can coordinate and stimulate distinct subsets of T helper (Th) cells, such as Th1 cells, to activate B cells for antibody secretion or Th2 cells to trigger cellular immunity-mediated cytotoxic T-cell responses ${ }^{11,12}$. The Ad vector can be delivered by different routes such as systemic or mucosal site administration, which makes vaccines convenient for immunization against respiratory pathogens that preferentially initiate infection at a mucosal site or in the respiratory tract $9,11,13$.

Here, we characterize the immunogenicity of recombinant $\mathrm{Ad}$ expressing $\mathrm{S}$ and S-engineered proteins of SARS-CoV-2 in animals. The immune responses induced by Ad vaccine mucosal immunization indicated significant Th1/Th2-biased immunity and a reduction in pulmonary viral loads and the associated inflammation induced by SARS-CoV-2 infection. The increased efficacy of the Ad vaccine against SARS-CoV-2 when administered intranasally rather than systemically was investigated. These results support the future development of mucosal Ad vaccines for clinical use to control SARS-CoV-2.

\section{Materials and Methods}

\section{Ethics statement}

The study was carried out in compliance with the ARRIVE guidelines. All animal experiments were conducted in accordance with the guidelines of the Laboratory Animal Center of the National Health Research Institutes (LAC-NHRI) in Taiwan. The animal use protocols were reviewed and approved by the NHRI Institutional Animal Care and Use Committee (Approval Protocol No. NHRI-IACUC-109073-M1-A-S01). To perform immunization or a viral challenge, the animals were placed in an anesthetic inhalator inhalation chamber containing isoflurane (initial phase: 5\%; maintenance phase: $3 \%-4 \%$ ) for $1 \mathrm{~min}$ before vaccine administration or SARS-CoV-2 challenge. 
After the investigation, the animals were euthanized by $100 \% \mathrm{CO}_{2}$ inhalation for 5 min followed by cervical dislocation to minimize suffering.

\section{Cell lines and viruses}

Human embryonic kidney cells (293A) were purchased from Invitrogen (Cat. R70507), and green monkey kidney cells (Vero) were purchased from the American Type Culture Collection (ATCC No. CCL-81). The 293 cells were cultured, grown and maintained in DMEM (HyClone, Cat. SH300) supplemented with $10 \%$ FBS in an incubator at $37^{\circ} \mathrm{C}$ with $5 \% \mathrm{CO}_{2}$. Vero cells were cultured, grown and maintained in M199 medium (GIBCO-BRL) supplemented with 5\% FBS in an incubator at $37^{\circ} \mathrm{C}$ with $5 \% \mathrm{CO}_{2}$. The SARS-CoV-2 strain (hCoV-19/Taiwan/4/2020) was isolated from the Taiwan Centers for Disease Control and propagated in Vero cells. The propagation of SARS-CoV-2 was performed in the P3-grade laboratory, which was maintained in accordance with the regulations and approved by the Taiwan CDC inspection service. The viral stocks were stored at $-80^{\circ} \mathrm{C}$. Viral stock titers were tested by cytopathic effect (CPE), and the TCID 50 values were calculated by Reed-Muench method.

\section{Animals, immunization, and live SARS-CoV2 challenge}

BALB/c mice and Syrian hamsters (purchased from the National Laboratory Animal Center, Taiwan) aged six to ten weeks were maintained in pathogen-free cages at the LAC-NHRI throughout the animal study. The BALB/c mice were primed with $1 \times 10^{7} \mathrm{pfu} / 50 \mu \mathrm{L}$ of Ad intranasally (i.n.) or subcutaneously (s.c.). After 14 days, the mice were boosted with the same dose of the respective immunogens administered via the same route. The mice were bled at 14 days and 1, 2, and 3 months after the booster immunization. The serum was analyzed by ELISA for the binding activity to recombinant S expressed in Sf9 insect cells (GenScript, Cat. 
Z0.481-100) and for neutralizing activity against SARS-CoV-2 by the neutralization$\mathrm{TCID}_{50}$ assay. For the challenge studies performed in the P3 laboratory, $1 \times 10^{5}$ $\mathrm{TCID}_{50}$ /hamster of live SARS-CoV-2 was administered i.n. 1 month after the second immunization. The hamsters were monitored for 6 days to record their body weights or sacrificed on days 3 and 6 after viral challenge, and the lung tissues were isolated for plaque assays and histochemistry.

Whole lungs were excised from the hamsters and homogenized, clarified, and titrated by the $\mathrm{TCID}_{50}$ on Vero cells in 96 -well plates. The cells were inoculated with serially diluted lung homogenate in M199 with 5\% FBS and incubated for 5 days. The cells were visualized when $50 \%$ were lysed by microscopy and used as sets of diluted lung homogenates according to the calculation of the virus titer in the sample.

For histochemistry, the lung tissues were placed directly into $10 \%$ formalin (Sigma-Aldrich) solution overnight and embedded in paraffin for sectioning. The sections were stained with $1 \% \mathrm{H} / \mathrm{E}$, and the pictures of each section were obtained at 100x and 200X magnification (Nikon DXM1200 CCD digital camera). The severity of inflammation in the $\mathrm{H} / \mathrm{E}$-stained sections was scored by following the reported criteria: grade 1: mixed mononuclear cell infiltrate next to the bronchus; grade 2: diffuse monocyte infiltration in alveolar spaces; grade 3: mixed diffuse infiltrates and small foci of mixed mononuclear cells (BALT); and grade 4: extensive dense collections of BALT and diffuse infiltrates ${ }^{14}$.

\section{Production of recombinant Ads}

AdCoV2 which encodes the condon-optimized SARS-CoV-2's S (AdCoV2-S) gene and transmembrane-deleted S (AdCoV2-SdTM) gene, the mutant S (AdCoV2-S(GA)) gene that has 2 amino acids mutated from $L Y$ to $G A$ at 611 and 612 residues of $S$, and

the condon-optimized SARS-CoV's S (AdSARS-S) gene, were cloned and generated 
with the pAd/CMVN5-DEST ${ }^{\mathrm{TM}}$ Gateway ${ }^{\circledR}$ Vector Kit (purchased from Cat. V49320, ThermoFisher Scientific) ${ }^{15,16}$. AdCoV2-S(GA) and AdSARS-S that are not related to this study are shown in the supplementary Figure 1. Ad-LacZ, which encodes the reporter gene bacterial $\beta$-galactosidase (LacZ), was also constructed as a control vector. The propagation of AdCoV2-S, AdCoV2-SdTM, and Ad-LacZ was performed in adherent $293 \mathrm{~A}$ cells in the presence of $10 \% \mathrm{FBS}^{9}$. Three days after Ad infection, cell pellets were harvested for freezing and thawing twice at $-80^{\circ} \mathrm{C}$ for $30 \mathrm{~min}$ and $37^{\circ} \mathrm{C}$ for $1 \mathrm{~min}$. The lysates were centrifuged at $3500 \mathrm{rpm}$ for $15 \mathrm{~min}$ at room temperature (RT), and the supernatants that contained Ad were collected. Recombinant Ad was purified and concentrated using Vivapure adenoPACK 100RT (Satorius Stedin Biotech). The purified viral titer was determined using a modified standard plaque assay ${ }^{9}$.

\section{Western blot}

Cellular protein lysate collects by Mem-PER ${ }^{\mathrm{TM}}$ Plus Membrane Protein Extraction Kit (Thermo Fisher Scientific, Cat. 89842). Ten micrograms of lysate from the Ad-infected cells was prepared and mixed with loading dye, loaded onto each well in a $10 \%$ SDS-polyacrylamide gel (SDS-PAGE; Amersham Biosciences-GE Healthcare, USA) and subjected to electrophoresis in 1x Tris-glycine SDS-running buffer ( $\mathrm{pH}$ 8.3). The SDS-PAGE gel was resolved, and the proteins in the gel were then transferred onto a nitrocellulose membrane (Hybond-ECL, Amersham Biosciences-GE Healthcare, USA). The membrane was soaked in $5 \%$ skim milk in $1 x$ PBS buffer ( $\mathrm{pH}$ 7.0) for $1 \mathrm{~h}$ at RT and then incubated with diluted SARS spike protein antibody, Mab5 (1:5000, a monoclonal antibody produced from a hybridoma line which was derived from the recombinant SARS'S S protein-immunized BALB/C 
mouse's spleen), SARS-CoV-2 (COVID-19, 2019-nCoV) spike antibody (1:3000; ProSci, Cat. 3525), beta-actin antibody (1:10000; Novus, Cat. NB600-501), or ATP1A1 monoclonal antibody (1:2000; Abnova, Cat. MAB2407) for 14 to $16 \mathrm{~h}$ at $4^{\circ} \mathrm{C}$. The membrane was subsequently washed with $1 x$ PBS plus $0.05 \%$ Tween 20 (PBS-T) followed by incubation with HRP-conjugated anti-rabbit antibody (1:10000; Genetex, Cat. GTX213110-01) or anti-mouse IgG antibody (1:10000; Thermo Fisher Scientific, Cat. After $1 \mathrm{~h}$ of incubation, the membrane was washed three times with PBS-T, the Millipore ECL substrate (Millipore, Cat. WBKLS0500) was layered onto the membrane, and then the signal was detected using an Amersham Imager 600.

\section{Collection of bronchoalveolar lavage (BAL)}

BAL fluids were collected by performing consecutive washes of the airspace of the lungs of individual experimental mice, with $1.0 \mathrm{~mL}$ of sterile PBS (pH 7.4). The samples obtained were stored in a $-80^{\circ} \mathrm{C}$ freezer until tested for their contents of specific proinflammatory cytokines.

\section{Enzyme-linked immunosorbent assay (ELISA)}

The SARS-CoV-2 spike protein (GenScript, Z03481-100) was coated onto a 96-well microplate at $50 \mathrm{ng}$ per well (Corning, Cat. 9018) overnight at $4^{\circ} \mathrm{C}$. The uncoated spike protein was removed, and then the wells were blocked with $200 \mu$ of blocking buffer (1X PBS with 1\% BSA) for $2 \mathrm{~h}$ at room temperature. The plates were washed with $200 \mu \mathrm{l}$ of PBS-T again, three times. Then, 10000-fold diluted HRP-conjugated donkey anti-mouse IgG, HRP-conjugated rabbit anti-mouse IgG1, and IgG2a antibodies (Invitrogen, Cat. 31430) were added to the wells and incubated 
for $1 \mathrm{~h}$. The plates were washed with $200 \mu$ l of PBST. TMB substrate (Seracare, Cat. 5120-0077) was used at $100 \mu \mathrm{l}$ per well and incubated for $10 \mathrm{~min}$ at room temperature. The reaction was halted with $100 \mu \mathrm{l}$ of $2 \mathrm{~N} \mathrm{H}_{2} \mathrm{SO}_{4}$. The optical density (O.D.) measurements were determined at $450 \mathrm{~nm}$ using a microplate reader. The endpoint serum dilution was calculated using the O.D. values and the cutoff value of serially diluted sera was set to twofold the background signal.

ELISA kit specific to mouse IFN- - , IL-1, IL-2, and IL-4 (ThermoFisher Scientific, Cat. 88-7314-88, 88-5019-88, 88-7024-88, and 88-7044-88, respectively) were used. 100 $\mu \mathrm{l} /$ well of capture antibodies specific to cytokine were added into 96-well plate and then incubate at $4^{\circ} \mathrm{C}$ for overnight. The plate was washed 3 times with $200 \mu \mathrm{l} /$ well PBS-T and then blocked with $200 \mu \mathrm{l} /$ well ELISA/ELISPOT Diluent (1X) Buffer at room temperature for 1 hour. After washing 3 times with PBS-T, $100 \mu \mathrm{l} /$ well of BAL fluid or the respective recombinant cytokine standard protein was added to wells and incubate at room temperature for 2 hours. The plate was washed again with PBS-T and added with $100 \mu \mathrm{l} /$ well the respective detection antibody to all wells and incubated at room temperature for 1 hour. The plate was washing again with PBS-T and $100 \mu \mathrm{l} /$ well of diluted Streptavidin-HRP was added and incubated at room temperature for 30 minutes. The plates were washed forth with PBS-T and added with TMB substrate to react for 15 minutes. Reaction was halted with stop solution and the O.D. measurements were determined at $450 \mathrm{~nm}$.

\section{Neutralizing assay}

One hundred microliters of serially diluted serum (reconstituted with preimmunized normal mouse serum to equal amounts of serum) was mixed with $200 \mathrm{TCID}_{50} / 100 \mu \mathrm{l}$ of SARS-CoV-2 and incubated for $2 \mathrm{~h}$ at $37^{\circ} \mathrm{C}$. The mixture was then added to $2.4 \times 10^{4}$ Vero cells in a 96-well plate, and each dilution was repeated 
four times. Vero cell cultures that had been treated with the same dose of virus without sera were used as a positive control. Five days later, the CPE of the cells was visualized by microscopy at the set point of the diluted-fold sera according to the calculation of the titer of neutralizing antibody possessed by the sera.

\section{Enzyme-linked immunosorbent spot assay (ELISPOT)}

RBC-free splenocytes $\left(5 \times 10^{5}\right)$ prepared from individual mice were seeded into each well of the 96-well filtration plates (Millipore, Cat. S2EM004M99) precoated with $0.5 \mu \mathrm{g} /$ well of monoclonal capture antibodies against murine IL-4 (BD, Cat. 551878) or IFN- $\gamma$ (BD, Cat. 551881), and then subjected to ELISPOT assays ${ }^{17}$. 3-amine-9-ethylcarbazole (AEC; Sigma-Aldrich, Cat. AEC101-KT) substrate was added to react for cytokine-specific immunospot generation. The generated spots were scored using an immunospot counting reader (Immunospot, Cellular Technology Ltd.). The obtained number of spots was the substrate to the number of spots gained from the respective well without restimulation (medium only). The results are expressed as the number of cytokine-secreting cells per $5 \times 10^{5}$ splenocytes seeded in the initial well, and the assay limit is higher than 1 cytokine-secreting cell per $5 \times$ $10^{5}$ splenocytes.

\section{Statistical analysis.}

One-way ANOVA test was used to analyze the results from Fig. 2, 3B, 4, 5, 6B, 7, and 8. Two-way ANOVA test was used to analyze the results from Fig. $3 \mathrm{~A}$ and $6 \mathrm{~A}$. The results were considered statistically significant when $P<0.05$. The symbols $*, * *$, and ***are used to indicate $P<0.05, P<0.01$, and $P<0.001$, respectively.

\section{Results}




\section{Expression of S and SdTM of SARS-CoV-2 in Ad-infected cells.}

We had constructed an Ad5 genome that carried the full-length and transmembrane-deleted S genes of the SARS-CoV-2, AdCoV2-S and AdCoV2-SdTM (Fig. 1A). To detect the expression of spike protein in Ad-infected cells, the immunoblotting of the culture medium and lysates with anti-S antibody was performed. The bands located at $\sim 185 \mathrm{kDa}$ and $\sim 182 \mathrm{kDa}$ corresponded to S- and SdTM-expressed proteins, respectively, and some degraded S proteins were detected in the cytosolic fraction of the lysates (Fig. 1B and supplementary Fig. 1A). S and SdTM were also detected in the membrane fraction of the lysate (Fig. 1C and supplementary Fig. 1B). However, only SdTM was detected in the Ad-infected culture medium (Fig. 1D and supplementary Fig. 1C). No signals were detected in any of the Ad-LacZ-infected samples (Fig. 1B, C, D). $\beta$-actin, ATP1A1, and mouse IgG heavy chain (supplementary Fig. 1A, 1B, and 1C, respectively) were detected independently as sample controls. These results indicated that either intact $\mathrm{S}$ or SdTM could be expressed in the respective Ad vector-infected cells. In addition, the membrane-deleted $\mathrm{S}$ that formed as a secretory protein was expected.

\section{Induction of SARS-CoV-2-specific antibody responses by AdCoV2 vaccines}

To investigate the immunogenicity of AdCoV2-S and AdCoV2-SdTM via systemic or mucosal administration, we immunized i.n. or s.c. BALB/c mice with $1 \times 10^{7}$ pfu of AdCoV2s on days 1 and 14 . The sera were serially collected at 2 weeks and 1,2 , and 3 months after the second immunization. The anti-SARS-CoV-2 IgG response and the binding titer in the sera were analyzed. The specific IgG antibody was significantly detected as early as 2 weeks (titer = approximately 12800 25600; Fig. 2A) and increased at one month (titer = approximately 204800; Fig. 2B), reaching its peak at the $2^{\text {nd }}$ month (titer $=>409600 ;$ Fig. $2 \mathrm{C}$ ) and then maintaining the antibody level at 
the $3^{\text {rd }}$ month (titer = approximately 409600; Fig. 2D) in AdCoV2-S-immunized i.n. serum. The antibody titers were approximately 3200, 25600, 102400, and 25600 at 2 weeks and 1, 2, and 3 months, respectively, in the AdCoV2-SdTM-immunized i.n. serum. Antibody titers of approximately 6400, 25600, 51200, and 25600 were detected in the AdCoV2-S-immunized s.c. serum at 2 weeks and 1, 2, and 3 months, respectively. No anti-SARS-CoV-2 IgG was detected at any of the time points in the Ad-LacZ-immunized serum (Fig. 2). In parallel, SARS-CoV-2-specific IgA was observed in the mucosal site of the vaginal wash $(\mathrm{vw})$, which was collected from the mice immunized i.n. with AdCoV2-S at 2 weeks and 1 month after the second immunization, compared to the lower level of SARS-CoV-2-specific IgA found in the vw obtained from the AdCoV2-SdTM-immunized i.n. mice. The vw from AdCoV2-S s.c. or Ad-LacZ i.n.-immunized mice did not have SARS-CoV-2-specific IgA (Fig. 3A).

A higher or lower antigen-specific $\lg G 1 / \lg G 2 a$ ratio in immunized sera that represented an immune response biased to Th2-mediated humoral or Th1-mediated cellular immunity, respectively, was reported ${ }^{18}$. SARS-CoV-2-specific IgG1 and IgG2a from all the AdCoV-2 vaccine sera were detected, in which the high to low sequence of IgG1 and IgG2a levels were AdCoV-2-S i.n. > AdCoV2-S s.c. > AdCoV2-SdTM-S i.n. (Fig. 3B). Interestingly, a higher IgG1 titer than that of IgG2a was observed in the i.n. groups administered AdCoV2-S and AdCoV2-SdTM, in contrast to the s.c. of AdCoV2-S, in which the IgG2a titer was higher than the titer of IgG1 (Fig. 3B). Therefore, the IgG1/IgG2a ratio from individual samples was calculated, and we found that AdCoV2-S i.n. induced a higher ratio score than AdCoV2-S s.c., but AdCoV2-S i.n. was similar to AdCoV2-SdTM i.n. (Fig. 3C). This finding reveals that different immunization routes might affect the immune reaction, whereas i.n. immunization favors the Th2 response, in contrast to Th1 responses, which are favored by s.c. immunization. 


\section{Induction of Th1/Th2-mediated cellular immunity by the AdCoV2 vaccine}

Cellular immunity is important for providing protective efficacy during the development of SARS-CoV-2 vaccines ${ }^{19}$. To examine which type of cellular immunity was induced by AdCoV2 vaccines, splenocytes were isolated from the Ad-immunized spleen at 2 months after the boost, followed by in vitro restimulation with medium only or recombinant S from SARS-CoV-2, and then assayed for cytokines by ELISPOT. S-stimulated lymphocytes from Ad-LacZ-immunized mice produced background IFN- $\gamma$ levels. By contrast, the highest IFN- $\gamma$ levels were detected in S-stimulated lymphocyte cultures from AdCoV2-S s.c.-immunized mice and moderate levels in AdCoV2-S i.n.- and AdCoV2-SdTM i.n.-immunized mice (Fig. 4A). Within the panel of Th2 cytokines that were assayed, IL-4 was most highly secreted by lymphocytes from AdCoV2-S s.c.-immunized mice compared to moderate levels in AdCoV2-S i.n.- and AdCoV2-SdTM i.n.-immunized mice. The background level of IL-4 secretion in Ad-LacZ-immunized mice was observed (Fig. 4B). The IFN- $/$ /IL-4 ratio was also calculated, and the ratio obtained from the AdCoV2-S i.n. group was equal to that of the AdCoV2-S s.c. group (Fig. 4C), thereby indicating that balanced Th1/Th2 responses were activated and that there was no difference in the activity needed to induce S-specific cellular immunity in response to AdCoV2-S i.n. and AdCoV2-S s.c. 


\section{Neutralizing antibody against SARS-CoV-2 induced by AdCoV2 immunization}

We further examined the SARS-CoV-2-specific neutralizing antibody induced by AdCoV2 immunization. Sera collected from AdCoV2-immunized i.n. or s.c. BALB/c mice at 2 weeks and 1 and 3 months after the second immunization were subjected to the SARS-CoV-2 neutralizing assay based on $\mathrm{TCID}_{50}$, which was described in the Materials and Methods. The neutralizing antibody was detected as early as 2 weeks later in serum from the AdCoV2-S and AdCoV2-SdTM groups, in which the titer of anti-SARS-CoV-2 antibody was approximately 192, 24, 120, and 0 (the limitation of $\mathrm{TCID}_{50}$ assay $=20$ ) in the serum from the AdCoV2-S i.n., AdCoV2-SdTM i.n., AdCoV2-S s.c., and vector control Ad-LacZ i.n. groups, respectively (Fig. 5A). After that, the neutralizing antibody was increased and the titers were approximately 576, 56, 144, and 0 in the 1-month serum from the AdCoV2-S i.n., AdCoV2-SdTM i.n., AdCoV2-S s.c., and Ad-LacZ i.n. groups, respectively (Fig. 5B). The titers were steadily increased to approximately $1088,64,144$, and 0 in the 3 -month serum from the AdCoV2-S i.n., AdCoV2-SdTM i.n., AdCoV2-S s.c., and Ad-LacZ i.n. groups, respectively (Fig. 5C). These results indicated that neutralizing antibodies against SARS-CoV-2 could be effectively induced and reached a peak at 3 months post vaccination with AdCoVs. The increase in protective antibodies elicits a long-term pattern in AdCoV2-immunized subjects. This finding is consistent with the SARS-CoV-2 binding activity results showing that AdCoV2-S possesses stronger immunogenicity than AdCoV-2-SdTM in inducing the anti-SARS-CoV-2 antibody response. In addition, the i.n. route is better than the s.c. route for AdCoV2 immunization.

\section{Potency of AdCoV2 vaccine in protection against SARS-CoV-2 infection}

A previous study showed that hamsters were an animal model for SARS-CoV and SARS-CoV-2 ${ }^{20}$. Therefore, we assessed the protective effect of AdCoV2 vaccines 
against SARS-CoV-2 in this animal model. Administration of AdCoV2-S or AdCoV2-SdTM via the i.n. route in hamsters following a challenge with live SARS-CoV-2 was performed. The animals were then sacrificed, and the pulmonary viral loads and lung tissue sections and histochemical staining were examined at days 3 and 6 post-viral challenge. On day 3 post-challenge, there was significant inhibition (10000 1000-fold reduction) of SARS-CoV-2 in the lungs of hamsters that were immunized with AdCoV2-S or AdCoV2-SdTM in comparison to Ad-LacZ-vaccinated tissues that contained high amounts of SARS-CoV-2 (Fig. 6A). Similarly, very little or no SARS-CoV-2 was detected in AdCoV2-S- and AdCoV2-SdTM-immunized lungs compared to some virus that was detected in Ad-LacZ-immunized lungs on day 6 post-challenge (Fig. 6B). Additionally, Ad-LacZ was not able to prevent SARS-CoV-2-induced weight loss, while AdCoV2-S and AdCoV2-SdTM could prevent severe weight loss in mice (Fig. 6C).

To evaluate the inhibition of SARS-CoV-2-induced lung inflammation and the safety of AdCoV2 vaccine administration in animals upon viral infection, we examined the lung pathogenesis of hamsters immunized with AdCoV2-S or AdCoV2-SdTM followed by SARS-CoV-2 infection. H/E-stained lung tissues from Ad-LacZ-immunized hamsters showed severe inflammation associated with lymphocyte infiltration among the alveoli, as expected. No or very mild inflammation was found in AdCoV-S-immunized tissues and mild to moderate inflammation was observed in AdCoV-SdTM-immunized tissues (Fig. 7A). We scored the degree of inflammation in the lungs of each visualized section. The sections from Ad-LacZ-immunized mice exhibited the highest score (average $=3.75$ ) for inflammation in the lung sections. By contrast, the lowest inflammation score (average $=0.5$ ) was observed in AdCoV-S-immunized tissues. A medium inflammation score (average $=3$ ) was found in AdCoV-SdTM-immunized tissues (Fig. 7A). These pathologic results correlated with 
the antibody response showing that AdCoV2-S i.n. was better than AdCoV2-SdTM i.n. at inducing and neutralizing antibodies. Additionally, AdCoV2-S was better than AdCoV2-SdTM at inhibiting SARS-CoV-2-induced lung inflammation in hamsters.

To further examine the induction of lung inflammation by AdCoV2 vaccine immunization, DMEM medium (Mock) or AdCoV2-immunized mice were sacrificed, and individual BALFs were prepared on 1 week after the two-dose immunization for detecting the secretion of proinflammatory cytokines. IFN- $\gamma$ was significantly induced in the BALF from AdCoV2-S in. and Ad-CoV2-SdTM i.n.-immunized groups but was not induced in the BALF from AdCoV2-S s.c. or Ad-LacZ i.n.. It indicates that i.n. delivery of AdCoV2 vaccine can induce Th1-biased responses in the lungs (Fig. 8A). IL-1, IL-2, and IL-4 (Fig. 8B) were measured and all of them were not induced after Ad vaccines immunization. The amounts of these cytokines were detected as background level as Mock group, suggesting that s.c. and i.n. of AdCoV2 vaccine did not induce the proinflammatory cytokines and was safe during the immunization in animals.

\section{Discussion}

Antibody-dependent enhancement (ADE) has been observed in coronaviruses, including SARS, MERS and other human respiratory virus infections, such as RSV and measles. Previous studies have shown that the induction of neutralizing antibodies, as well as pathogen-specific cellular immunity against coronavirus infection, are important for effective vaccine development. SARS-CoV prototype vaccines that induce a Th2-type immune response have a risk of ADE in animal models ${ }^{21,22}$. The WHO has noticed that Th1-mediated immunity and neutralizing antibody responses are the key factors needed for success against SARS-CoV-2 when applied to the development of candidate vaccines ${ }^{23}$. Thus, there is a suggested risk of ADE in SARS-CoV-2 vaccines and antibody-based interventions that should receive attention 
24. Recombinant replication-incompetent adenoviruses have an acceptable safety profile in humans and are able to induce neutralizing antibodies, CD4 and CD8 T cell responses and a Th1-biased immune response in animals and humans ${ }^{25-28}$. Our studies also showed that AdCoV2s elicit strong antibody response (eg., AdCoV2-S; Fig. 2) and active the Th1 and Th2-comparable cellular immunity (eg., IFN- $\gamma$ and II-4 release respectively, shown in Fig. 4, and IgG2a and IgG1 induction, shown in Fig. 4) in response to AdCoV2-S and AdCoV2-SdTM. Indeed, AdCoV2-SdTM induced a weaker neutralizing antibody response than AdCoV2-S (Fig. 5). The intact S protein expressed by AdCoV2-S-infected cells might highly mimic the prefusion form of $\mathrm{S}$ protein on the viral particle that could activate an adequate host antibody to recognize the natural virus. In other words, the structure could be changed after the deletion of the membrane domain from the $\mathrm{S}$ protein, which might induce inadequate antibodies and subsequently affect binding to the virus. Second, the virus-binding titer and neutralizing antibody titer in the serum after i.n. AdCoV2-S administration was higher than that the serum after s.c. AdCoV2-S administration (Fig. 2 and Fig. 5, respectively). These findings indicated that mucosal administration (i.n.) of Ad-based vaccines is more immunogenic than systemic administration (s.c.), especially for respiratory infections. In fact, mucosal delivery of AdCoV2-S induced the dominant $\lg G$ and $\lg A$, compared to the systemic administration of AdCoV2-S which induced IgG only, which are important for neutralizing pathogens in the respiratory tract during the early stage of infection. An Ad5-encoded influenza $A / P R / 8 / 34$ hemagglutinin $(H A)$ with an i.n. or epicutaneous injection route in humans was found to be safe and immunogenic for inducing the anti-HA antibodies. The intranasal vaccine of Ad-PR.8.HA was more potent than when administered epicutaneously ${ }^{29}$. However, the third event in this study reveals that AdCoV2-S administration via the i.n. or s.c. route, or AdCoV2-SdTM via the i.n. route, all 
showed sufficient efficacy in preventing SARS-CoV-2 infection, as evidenced by the effect on hamsters that were immunized following challenge with SARS-CoV-2 (Fig. 6). This result demonstrated that not only neutralizing antibodies but also Th1/Th2-mediated cellular immunity contribute to inhibiting SARS-CoV-2 in the host.

Several studies have shown that mucosal but not systemic immunization with an Ad vaccine induced long-term immunity against pathogens, such as the Ad vector-expressing glycoprotein B of herpes simplex virus type 2 (HSV-2) against HSV-2 30. Long-lasting anti-respiratory syncytia virus (RSV) immunity was induced by a nasal Ad vector expressing the fusion protein Ad-RSV-F ${ }^{31}$. Our results also confirmed that the neutralizing antibody against SARS-CoV-2 induced by i.n. delivery of AdCoV2-S was even continuingly increased up to highest titer after three months of the second booster (Fig. 5).

Pre-existing anti-Ad host immunity is always an issue to track when assessing the efficacy of Ad-based vaccines. A high titer of anti-influenza $\mathrm{HI}$ antibody was induced in subjects who had a high pre-existing antibody titer to Ad by Ad-PR.8 HA vaccine immunization. This finding indicated that the Ad-PR.8. HA vaccine showed no correlation with pre-existing neutralizing antibodies to Ad and the potency of Ad vaccines in a human trial ${ }^{29}$. Nasal injection of an Ad5-expressing Ebola Zaire glycoprotein (Ad5-ZGP), which bypassed the influence of the pre-existing anti-Ad antibody, fully protected mice from a lethal challenge with Ebola ${ }^{32}$. The anti-Ad antibody did not influence either anti-RSV antibody induction by Ad-RSV-F via i.n. immunization or the induction of protection against RSV challenge in mice ${ }^{9}$. Thus, intranasal delivery of the AdCoV2 vaccine has the advantage of expressing full protection against SARS-CoV-2.

There were clinical observations of COVID-19 patients who had severe inflammatory responses in the lungs corresponding to viral burdens in the pulmonary 
epithelial cells and resulting in respiratory failure ${ }^{33}$. This finding also correlated with our result that pulmonary inflammation was recapitulated by Ad-LacZ immunization following a challenge with SARS-CoV-2 in animals. No or very mild inflammation in the lungs of mice that received AdCoV2-S was observed (Fig. 7). This consequence is corresponding to the Th1 cytokine and no proinflammatroy cytokine secretions in the lungs of mice which received AdCoV2-S i.n. (Fig. 8).

Collectively, this evidence indicates that the protective immunogenicity of AdCoV2 is potent against SARS-CoV-2 infections. AdCoV2 administered through different routes of injection might induce different immune characteristics; mucosal vs. systemic immune responses, all showed that they can induce protective immune responses against SARS-CoV-2, while only i.n. delivery elicits the prevention of virus-induced lung inflammation and no vaccine-enhanced disease effects and prompts us to conduct further human trials.

\section{Conflict of interest}

All the authors of this manuscript state that there are no conflicts of interest.

\section{Acknowledgments}

We thank the Centers for Disease Control, Ministry of Health and Welfare for providing SARS-CoV-2 virus and all staff of ABSL3 team of NHRI who operate the virus in this study. This work was supported by grants (MOST 109-2311-B-400-001-) from the Taiwan Ministry of Science and Technology (http://www.most.gov.tw/mp.aspx). Nai-Hsiang Chung carried out his thesis research under the auspices of the Graduate Program of Biotechnology in Medicine, NTHU and NHRI.

\section{References}

1 Christian Drosten, S. G., Wolfgang Preiser, Sylvie van der Werf, Hans-Reinhard Brodt, Stephan Becker, Holger Rabenau, Marcus Panning, Larissa Kolesnikova, 
Ron A M Fouchier, Annemarie Berger, Ana-Maria Burguière, Jindrich Cinatl, Markus Eickmann, Nicolas Escriou, Klaus Grywna, Stefanie Kramme, Jean-Claude Manuguerra, Stefanie Müller, Volker Rickerts, Martin Stürmer, Simon Vieth, Hans-Dieter Klenk, Albert D M E Osterhaus, Herbert Schmitz, Hans Wilhelm Doerr. Identification of a novel coronavirus in patients with severe acute respiratory syndrome. The New England journal of medicine 348, 1967-1976 (2003).

2 Raoul J. de Groot, S. C. B., Ralph S. Baric, Caroline S. Brown, Christian Drosten, Luis Enjuanes, Ron A. M. Fouchier, Monica Galiano, Alexander E. Gorbalenya, Ziad A. Memish, Stanley Perlman, Leo L. M. Poon, Eric J. Snijder, Gwen M. Stephens, Patrick C. Y. Woo, Ali M. Zaki, Maria Zambon, John Ziebuhr. Middle East Respiratory Syndrome Coronavirus (MERS-CoV): Announcement of the Coronavirus Study Group. Journal of virology 87 7790-7792 (2013).

3 Jessie Huang, A. J. H., Kristine M. Abo, Rhiannon B. Werder, Carlos Villacorta-Martin, Konstantinos-Dionysios Alysandratos, Mary Lou Beermann, Chantelle Simone-Roach, Jonathan Lindstrom-Vautrin, Judith Olejnik, Ellen L. Suder, Esther Bullitt, Anne Hinds, Arjun Sharma, Markus Bosmann, Ruobing Wang, Finn Hawkins, Eric J. Burks, Mohsan Saeed, Andrew A. Wilson, Elke Muhlberger, and Darrell N. Kotton. SARS-CoV-2 Infection of Pluripotent Stem CellDerived Human Lung Alveolar Type 2 Cells Elicits a Rapid Epithelial-Intrinsic Inflammatory Response. Cell Stem Cell 27, 1-12 (2020).

4 Zarbock, S. L. a. A. Coronaviruses and SARS-CoV-2: A Brief Overview. ANESTHESIA \& ANALGESI, 1-4, doi:10.1213/ANE.0000000000004845 (2020).

5 Alexandra C. Walls, Y.-J. P., M. Alejandra Tortorici, Abigail Wall, Andrew T. McGuire, and David Veesler. Structure, Function, and Antigenicity of the SARS-CoV-2 Spike Glycoprotein. Cell 180 281-292 (2020).

6 Robert N. Kirchdoerfer, C. A. C., Nianshuang Wang, Jesper Pallesen, Hadi M. Yassine, Hannah L. Turner, Kizzmekia S. Corbett, Barney S. Graham, Jason S. McLellan \& Andrew B. Ward. Pre-fusion structure of a human coronavirus spike protein. Nature 531 118-121, doi:10.1038/nature17200 (2016).

7 Li, F. Structure, Function, and Evolution of Coronavirus Spike Proteins. Annual Review of Virology 3, 237-261, doi:10.1146/annurev-virology-110615-042301 (2016).

8 Markus Hoffmann, H. K.-W., Simon Schroeder, Nadine Krü ger, Tanja Herrler, Sandra Erichsen, Tobias S. Schiergens, Georg Herrler, Nai-Huei Wu, Andreas Nitsche, Marcel A. Mü Iler, Christian Drosten,3 and Stefan Pöhlmann. SARS-CoV-2 Cell Entry Depends on ACE2 andTMPRSS2 and Is Blocked by a Clinically ProvenProtease Inhibitor. Cell 81, 271-280 (2020). 
9 Shao, H. Y. et al. Immunogenic properties of RSV-B1 fusion (F) protein gene-encoding recombinant adenoviruses. Vaccine, doi:S0264-410X(09)00992-X [pii]

10.1016/j.vaccine.2009.07.004 (2009).

10 Kohlmann, R. et al. Protective efficacy and immunogenicity of an adenoviral vector vaccine encoding the codon-optimized $\mathrm{F}$ protein of respiratory syncytial virus. J Virol 83, 12601-12610, doi:10.1128/JVI.01036-09 (2009).

11 Coughlan, L., Mullarkey, C. \& Gilbert, S. Adenoviral vectors as novel vaccines for influenza. The Journal of pharmacy and pharmacology 67, 382-399, doi:10.1111/jphp.12350 (2015).

12 Barouch, D. H. \& Nabel, G. J. Adenovirus vector-based vaccines for human immunodeficiency virus type 1 . Hum Gene Ther 16, 149-156, doi:10.1089/hum.2005.16.149 (2005).

13 Wang, J. et al. Single mucosal, but not parenteral, immunization with recombinant adenoviral-based vaccine provides potent protection from pulmonary tuberculosis. J Immunol 173, 6357-6365 (2004).

14 Brincks, E. L. et al. Antigen-specific memory regulatory CD4+Foxp3+ T cells control memory responses to influenza virus infection. J Immunol 190, 3438-3446, doi:10.4049/jimmunol.1203140 (2013).

15 Kossila, M. et al. Improvement in adenoviral gene transfer efficiency after preincubation at +37 degrees $C$ in vitro and in vivo. Mol Ther $5,87-93$, doi:10.1006/mthe.2001.0516 (2002).

16 Russell, W. C. Update on adenovirus and its vectors. J Gen Virol 81, 2573-2604, doi:10.1099/0022-1317-81-11-2573 (2000).

17 Shao, H. Y. et al. Immunoprotectivity of HLA-A2 CTL peptides derived from respiratory syncytial virus fusion protein in HLA-A2 transgenic mouse. PloS one 6, e25500, doi:10.1371/journal.pone.0025500 (2011).

18 Holdsworth, S. R., Kitching, A. R. \& Tipping, P. G. Th1 and Th2 T helper cell subsets affect patterns of injury and outcomes in glomerulonephritis. Kidney international 55, 1198-1216, doi:10.1046/j.1523-1755.1999.00369.x (1999).

19 Gregory A Poland, I. G. O., Richard B Kennedy. SARS-CoV-2 immunity: review and applications to phase 3 vaccine candidates. The lancet 396 1595-1606 (2020).

20 Imai, M. et al. Syrian hamsters as a small animal model for SARS-CoV-2 infection and countermeasure development. Proceedings of the National Academy of Sciences of the United States of America 117, 16587-16595, doi:10.1073/pnas.2009799117 (2020).

21 Honda-Okubo, Y. et al. Severe acute respiratory syndrome-associated 
coronavirus vaccines formulated with delta inulin adjuvants provide enhanced protection while ameliorating lung eosinophilic immunopathology. Journal of virology 89, 2995-3007, doi:10.1128/JVI.02980-14 (2015).

22 Tseng, C. T. et al. Immunization with SARS coronavirus vaccines leads to pulmonary immunopathology on challenge with the SARS virus. PloS one 7, e35421, doi:10.1371/journal.pone.0035421 (2012).

23 Kathryn M Edwards, W. A. O. Coronavirus disease 2019 (COVID-19): Vaccines to prevent SARS-CoV-2 infection. UpToDate (2020).

24 Lee, W. S., Wheatley, A. K., Kent, S. J. \& DeKosky, B. J. Antibody-dependent enhancement and SARS-CoV-2 vaccines and therapies. Nature microbiology 5, 1185-1191, doi:10.1038/s41564-020-00789-5 (2020).

25 Anywaine, Z. et al. Safety and Immunogenicity of a 2-Dose Heterologous Vaccination Regimen With Ad26.ZEBOV and MVA-BN-Filo Ebola Vaccines: 12-Month Data From a Phase 1 Randomized Clinical Trial in Uganda and Tanzania. The Journal of infectious diseases 220, 46-56, doi:10.1093/infdis/jiz070 (2019).

26 Baden, L. R. et al. Assessment of the Safety and Immunogenicity of 2 Novel Vaccine Platforms for HIV-1 Prevention: A Randomized Trial. Annals of internal medicine 164, 313-322, doi:10.7326/M15-0880 (2016).

27 Barouch, D. H. et al. Evaluation of a mosaic HIV-1 vaccine in a multicentre, randomised, double-blind, placebo-controlled, phase 1/2a clinical trial (APPROACH) and in rhesus monkeys (NHP 13-19). Lancet 392, 232-243, doi:10.1016/S0140-6736(18)31364-3 (2018).

28 Shukarev, G., Callendret, B., Luhn, K., Douoguih, M. \& consortium, E. A two-dose heterologous prime-boost vaccine regimen eliciting sustained immune responses to Ebola Zaire could support a preventive strategy for future outbreaks. Human vaccines \& immunotherapeutics 13, 266-270, doi:10.1080/21645515.2017.1264755 (2017).

29 Van Kampen, K. R. et al. Safety and immunogenicity of adenovirus-vectored nasal and epicutaneous influenza vaccines in humans. Vaccine 23, 1029-1036, doi:10.1016/j.vaccine.2004.07.043 (2005).

30 Gallichan, W. S. \& Rosenthal, K. L. Long-term immunity and protection against herpes simplex virus type 2 in the murine female genital tract after mucosal but not systemic immunization. J Infect Dis 177, 1155-1161 (1998).

31 Shao, H. Y. et al. Immunogenicity of an adeno-vector vaccine expressing the $F$ protein of a respiratory syncytial virus manufactured from serum-free suspension culture. Antiviral research 130, 27-35, doi:10.1016/j.antiviral.2016.03.011 (2016). 
32 Croyle, M. A. et al. Nasal delivery of an adenovirus-based vaccine bypasses pre-existing immunity to the vaccine carrier and improves the immune response in mice. PloS one 3, e3548, doi:10.1371/journal.pone.0003548 (2008).

33 Schaefer, I. M. et al. In situ detection of SARS-CoV-2 in lungs and airways of patients with COVID-19. Modern pathology : an official journal of the United States and Canadian Academy of Pathology, Inc 33, 2104-2114, doi:10.1038/s41379-020-0595-z (2020).

\section{Author Contribution:}

Yen-Hung Chow, conceived and designed the whole experiments, analyzed the data, and wrote the main manuscript text. Nai-Hsiang Chung, performed the experiments to obtain Fig. 1, 2, 3, 4, 5, and 8. Ying-Chin Chen, helped to perform animal study to obtain Fig. 2, 3, and 4, and cultured SARS-CoV2 used in whole experiments. Shiu-Ju Yang, Yu-Ching Lin, and Horng-Yunn Dou, performed the experiments to obtain Fig. 6 and 7. Ching-Len Liao, advised the study direction and provided the materials/reagent for SARS-CoV2 culture. All authors reviewed the manuscript.

\section{Figure legends:}

Figure 1. Construction and analysis of SARS-CoV-2 spike expression in AdCoV2 vaccines

AdCoV2-S and AdCoV2-SdTM were constructed as described in the Materials and Methods, and their brief genetic maps are shown (A). Uninfected 293 cells (mock) and cells infected individually with Ad-LacZ, AdCoV2-S, and AdCoV2-SdTM at an MOI of 0.1 for $24 \mathrm{~h}$ were used. Parts of the culture medium (D) and the lysate were harvested. Lysates were extracted to obtain cytosolic (B) and membrane (C) fractions as described in the Materials and Methods. The prepared samples ( $B, C$, and $D)$ were 
mobilized in SDS-PAGE electrophoresis and antigenically transferred onto the western blot membrane, then blotted with S-specific antibodies, (B and C) Mab5 or (D) COVID-19, 2019-nCoV. The target proteins marked by arrow were shown. Specific antibodies against $\beta$-actin, ATP1A1, and mouse IgG heavy chain were used as sample controls for the immunoblots of the cytosolic fraction, membrane fraction, and culture medium, respectively. The data are representative of the results derived from two independent experiments.

Figure 2. Induction of SARS-CoV-2-specific IgG by AdCoV2 vaccines.

BALB/c mice were immunized with $1 \times 10^{7}$ pfu Ad-LacZ, AdCoV2-S, or AdCoV2-SdTM via i.n. or AdCoV2-S via s.c. at 14-day intervals. The varying dilutions of sera collected at 14 days (A) or 1 (B), 2 (C), or 3 (D) months post boost were analyzed using IgG anti-recombinant intact $S$ protein-immobilized ELISA. Anti-mouse IgG conjugated with HRP was used as the detection antibody. The titer of specific antibody in the serum was calculated as a fold dilution of the tested sera, whose detected value at OD450 $\mathrm{nm}$ of absorbance $>2$ times the value at OD450 $\mathrm{nm}$ of absorbance obtained from the medium alone. A one-way ANOVA test was used.

Figure 3. Induction of the anti-SARS-CoV-2 IgG subclass by AdCoV2 vaccines.

Sera from BALB/c mice immunized twice with $1 \times 10^{7}$ pfu Ad-LacZ, AdCoV2-S, or AdCoV2-SdTM via i.n. or AdCoV2-S via s.c. at 14-day intervals were set to detect the subclass of specific Ig. For (A) IgA detection, vw were collected at 14 days or 1 month after the second immunization, and $1 / 10$ or $1 / 40$-diluted individual vws were subjected to recombinant intact $S$ protein-immobilized ELISA. For SARS-CoV-2-specific (B) IgG1 IgG2a detection, diluted immune sera were examined using recombinant intact $S$ protein-immobilized ELISA. Anti-mouse $\lg G 1$ or $\lg G 2 a$ 
conjugated with HRP was used as the detected antibody. (C) The ratio of IgG1/IgG2a based on the titer obtained from (B) was calculated. Similar results from 2 independent experiments were obtained, and one of the results is shown. One and two-way ANOVA test was used.

Figure 4. Induction of SARS-CoV-2-specific cellular immunity by AdCoV2 vaccines. Five mice per group of BALB/c mice were individually primed and boosted at 14-day intervals though i.n. or s.c. routes with $1 \times 10^{7}$ pfu of AdCoV2 or Ad-LacZ. Ad-immunized mice were sacrificed 3 months after the vaccine boost, and splenocytes were collected and cultured in the presence or absence of $10 \mu \mathrm{g} / \mathrm{mL} \mathrm{S}$ protein or $10 \mu \mathrm{g} / \mathrm{ml}$ Con $\mathrm{A}$. A total of $5 \times 10^{5}$ splenocytes were seeded on (A) anti-IFN-y or (B) anti-IL-4 capture antibody-coated ELISPOT plates for 24 or $48 \mathrm{~h}$, respectively, for the ELISPOT assay. Cytokine-positive spots were developed, and the results are expressed as the number of spots +/- two standard deviations for each experimental group. (C) The ratio of IFN- - -positive spot numbers/IL-4-positive spot numbers was calculated and shown. A one-way ANOVA test was used.

Figure 5. Induction of neutralizing antibody against SARS-CoV-2 by AdCoV2 vaccines.

Sera collected from AdCoV vaccine-immunized BALB/c mice at 14 days (A), 1 month (B), and 3 months (C) post-immunization as described in the legend of Fig. 2 were applied to the neutralizing assay, which is described in the Materials and Methods. The experimental protocol was followed for the regulation of the RG-3 level during laboratory operation. One-way ANOVA test was used.

Figure 6. AdCoV2 vaccines protect animals from SARS-CoV-2 infection. 
Four adult hamsters per group were preimmunized twice i.n. with $3 \times 10^{7} \mathrm{pfu} \mathrm{Ad}-\mathrm{LacZ}$, AdCoV2-S, or AdCoV2-SdTM over a 14-day interval and then infected i.n. with $1 \times 10^{5}$ pfu of live SARS-CoV-2 1 month after the second vaccine shot. (A) On days 3 and (B) 6 post-infection, the hamsters were sacrificed and the lung tissues were homogenized for the $\mathrm{TCID}_{50}$ assay. One and two-way ANOVA test were used for statistical analysis. (C) The body weight of AdCoV2-immunized hamsters followed by SARS-CoV-2 challenge was recorded. When Ad-LacZ i.n. compares with AdCoV2-S i.n., the symbols * are used to indicate $\mathrm{P}<0.05$. When Ad-LacZ i.n. compares with AdCoV2-SdTM i.n., the symbols \# are used to indicate $P<0.05$. Two-way ANOVA test were used for statistical analysis.

\section{Figure 7. Inhibition of lung inflammation induced in SARS-CoV-2-challenged hamsters by AdCoV2 vaccines.}

Sections of lung tissues collected from AdCoV vaccine-immunized hamsters on day 6 post-infection as described in the legend of Fig. 6 were stained with H/E dye. (A) A representation of the pictures of $\mathrm{H} / \mathrm{E}$-stained lung sections from each hamster is shown and the score of disease as judge by the criteria as described in the Materials and Methods is marked. (B) The severity of the histopathology in each section is graded and shown based upon the score. One-way ANOVA test were used for statistical analysis.

\section{Figure 8. Detection of proinflammatory cytokines in the AdCoV2-immunized BALF}

BALF from individual mice immunized twice via i.n. route with Ad-LacZ, AdCoV2-S, or AdCoV2-SdTM, or s.c. route with AdCoV2-S, were quantitated for their contents of (A) IFN- $\gamma$ and (B) IL-1, IL-2 and IL-4, using the reagents and protocols that were described in the Material and Methods. Results are presented as the concentration of the 
cytokine in pg per $\mathrm{mL}$. One-way ANOVA test were used for statistical analysis. 
Figures

Figure 1

A

Ad-LacZ

AdCoV2-S

AdCoV2-SdTM

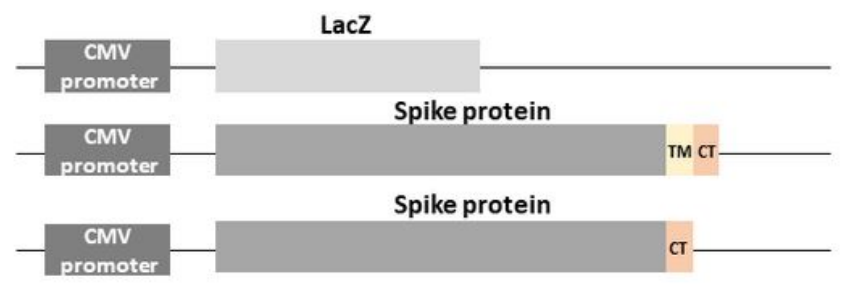

B

C

Membrane fraction

D

\section{Culture medium}
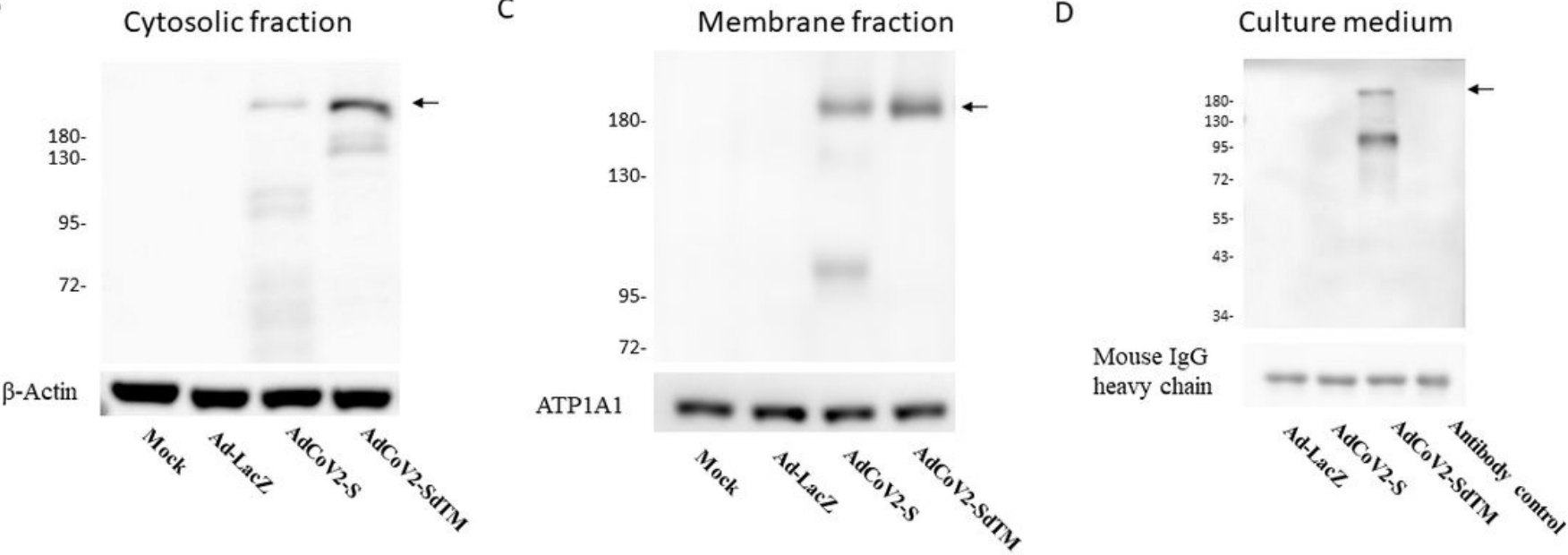

\section{Figure 1}

"See the Supplemental Files section for the complete figure caption". 
A
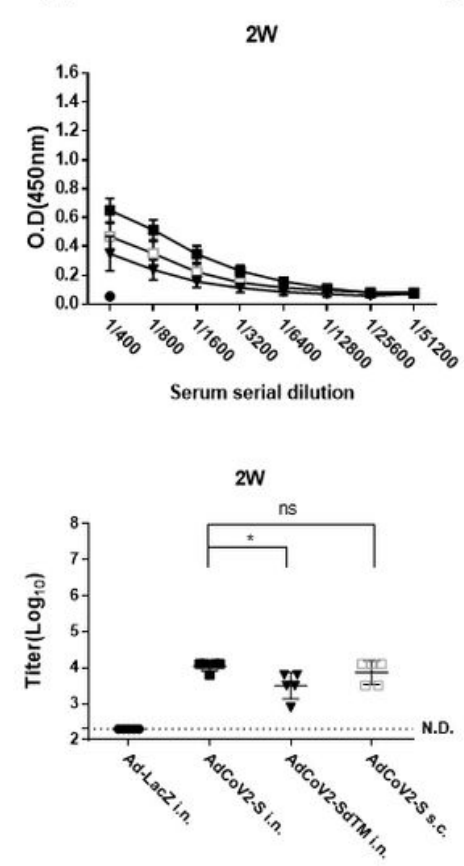

B
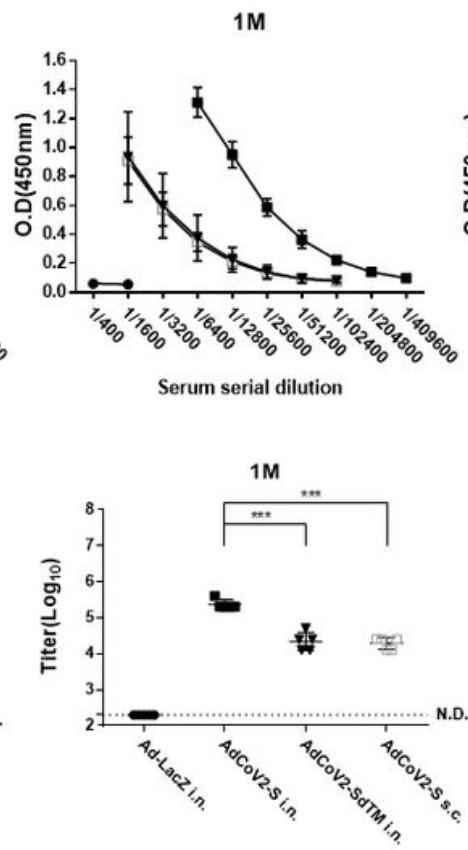

C
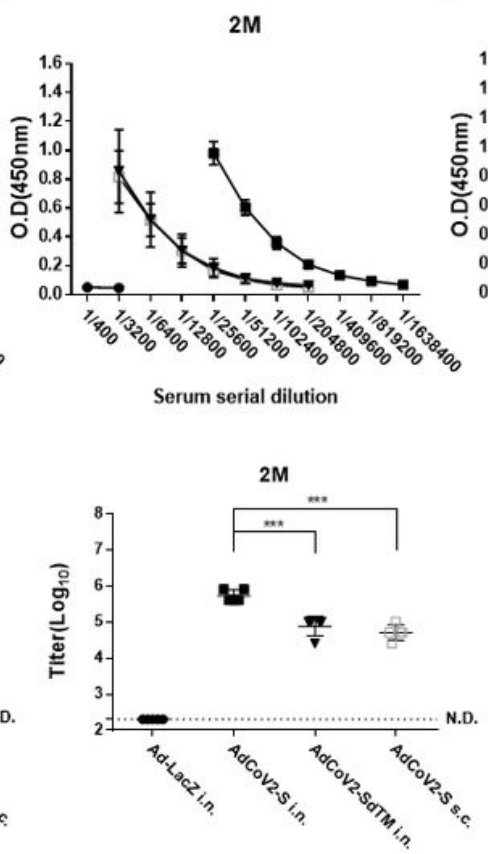

D

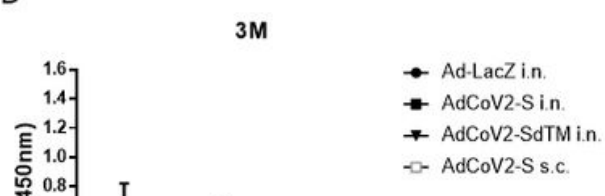

\section{Figure 2}

"See the Supplemental Files section for the complete figure caption".

Figure 3

A

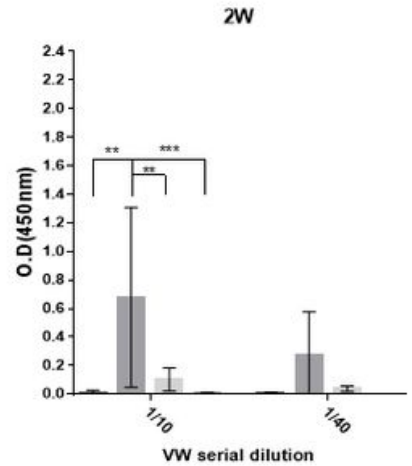

B

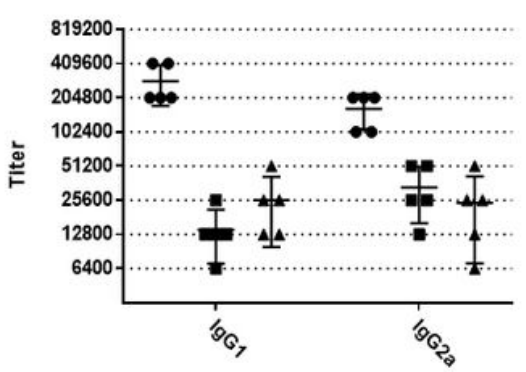

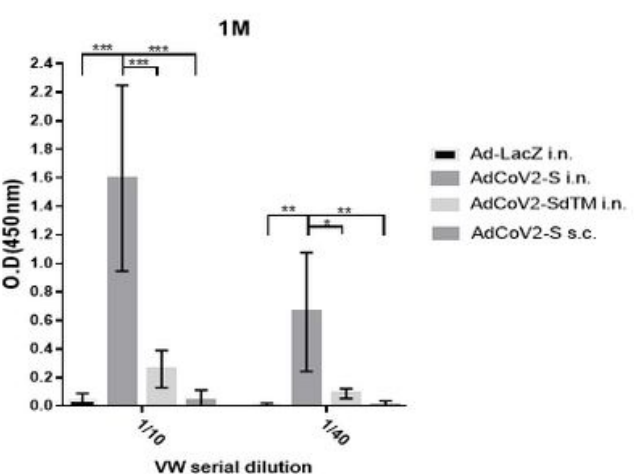

C

- AdCoV2-Sin.

- AdCovz-S s.c.

- AdCoV2-SdTMi.n

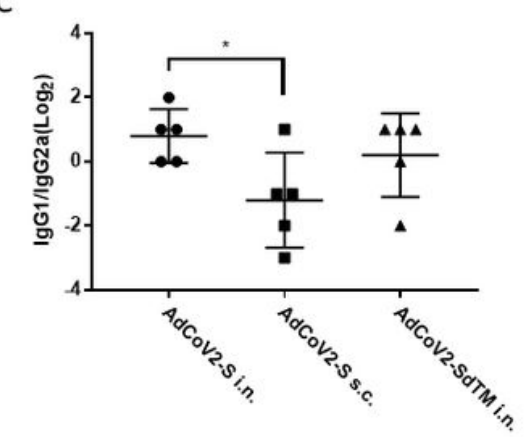


Figure 3

"See the Supplemental Files section for the complete figure caption".

Figure 4

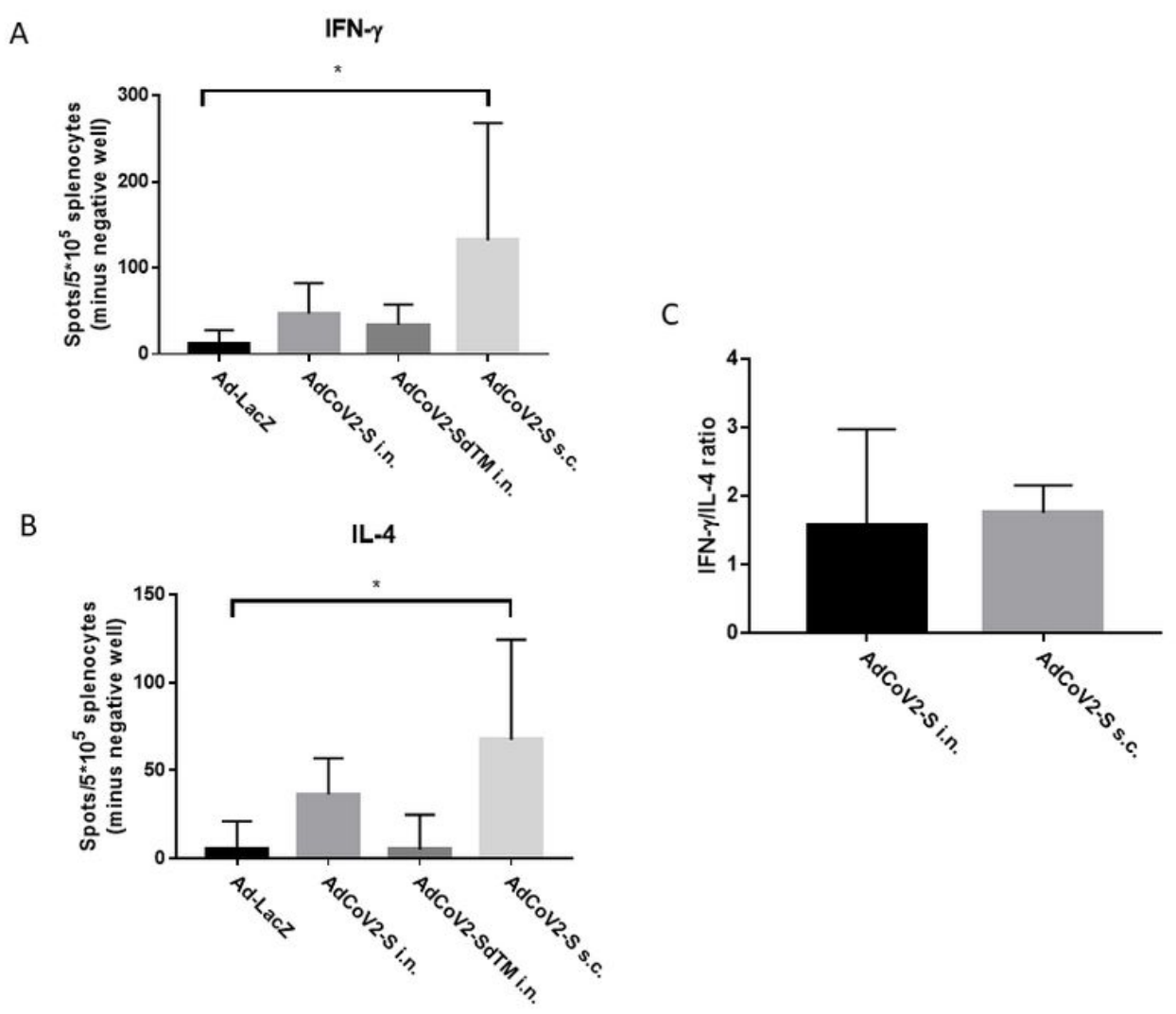

Figure 4

"See the Supplemental Files section for the complete figure caption". 
Figure 5
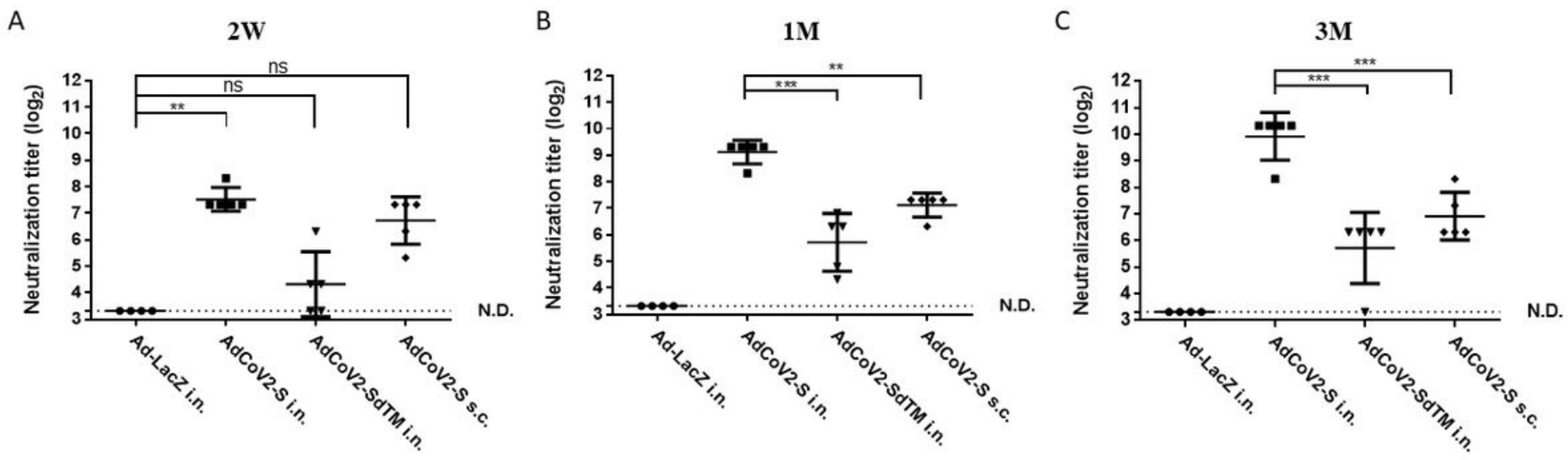

\section{Figure 5}

"See the Supplemental Files section for the complete figure caption".

Figure 6

A

3 days post-challenge

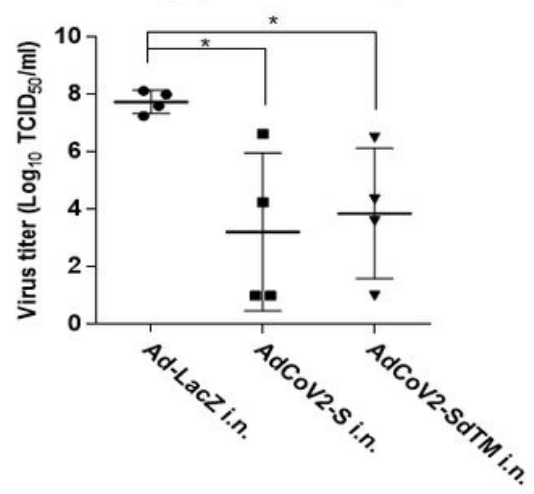

C
B

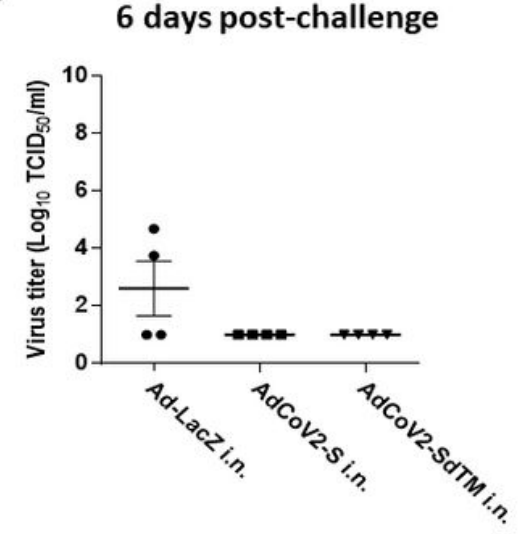

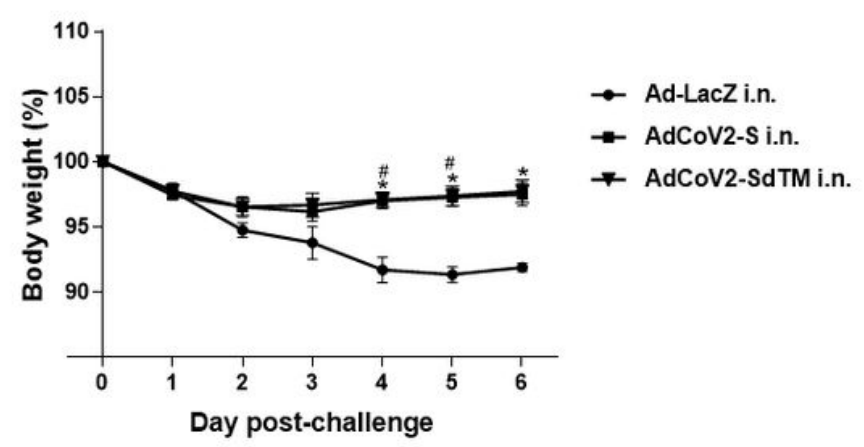


Figure 6

"See the Supplemental Files section for the complete figure caption".

Figure 7

A
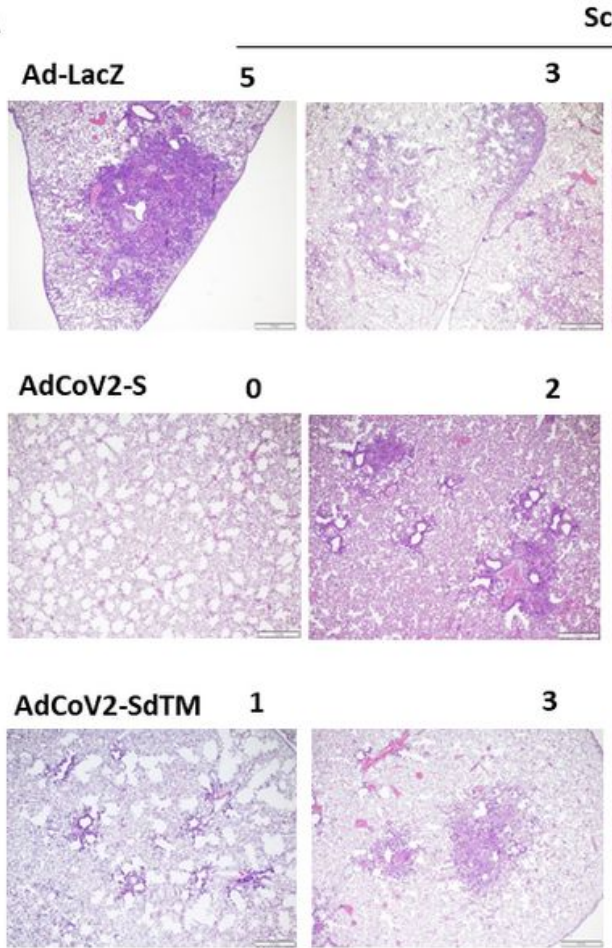

Scale bar: $500 \mu \mathrm{m}$

3
3
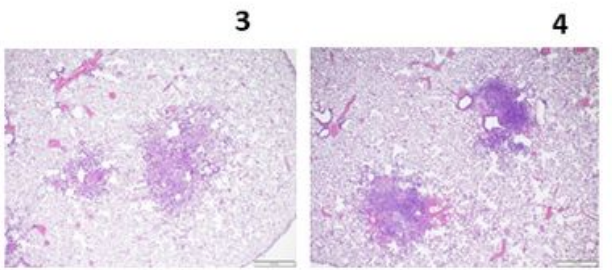

4
Score
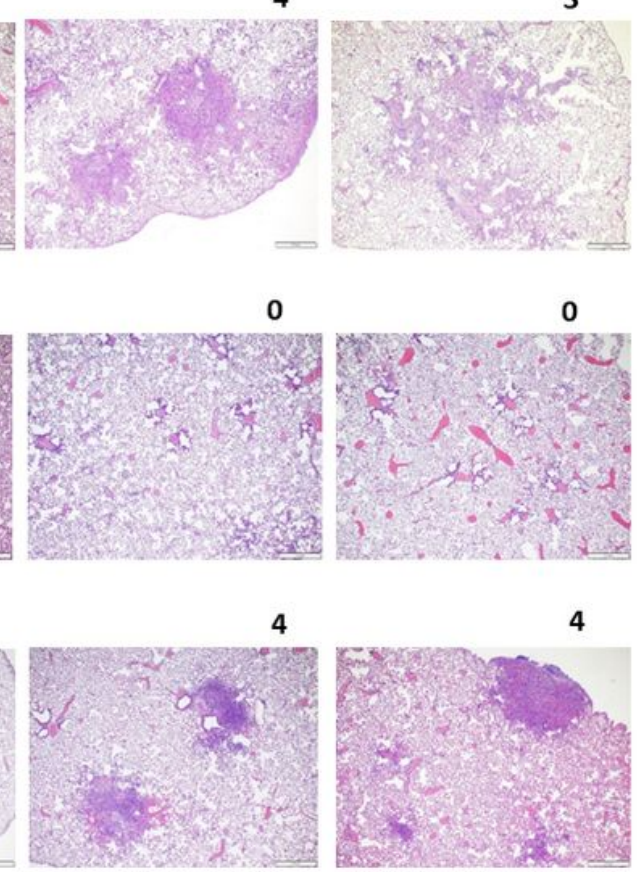

0

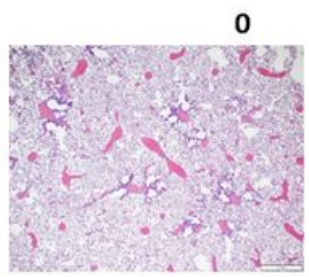

\section{Figure 7}

"See the Supplemental Files section for the complete figure caption".

B

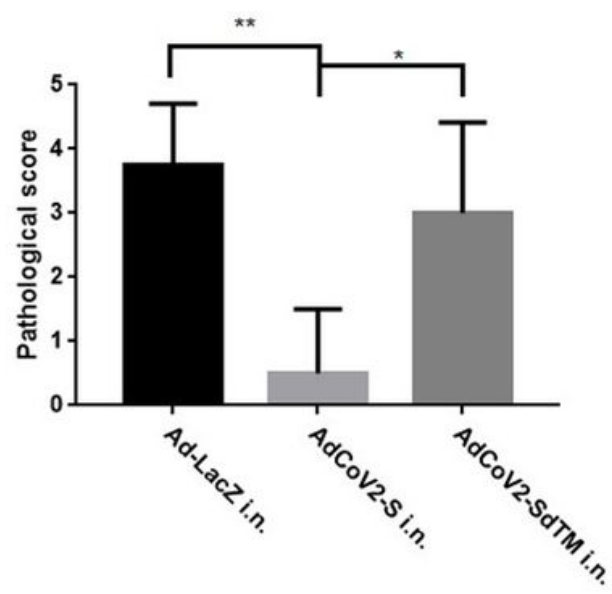


Figure 8

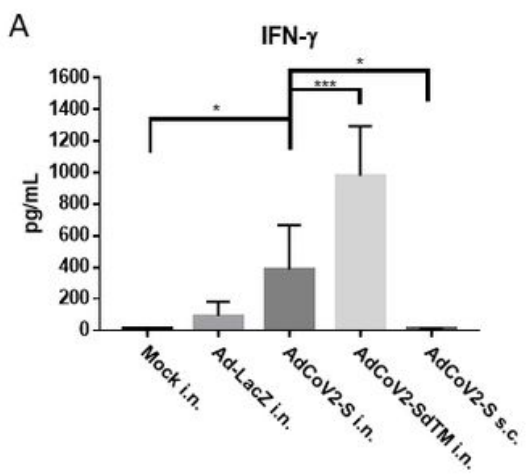

B

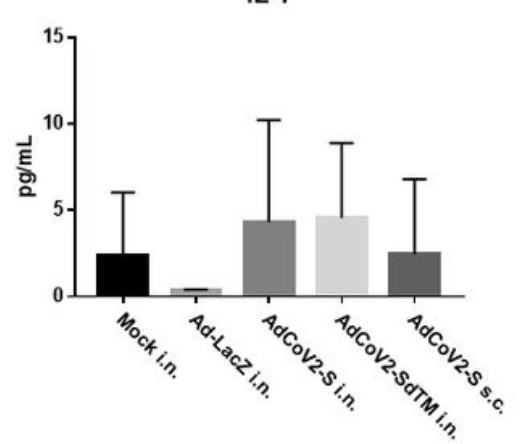

IL-2

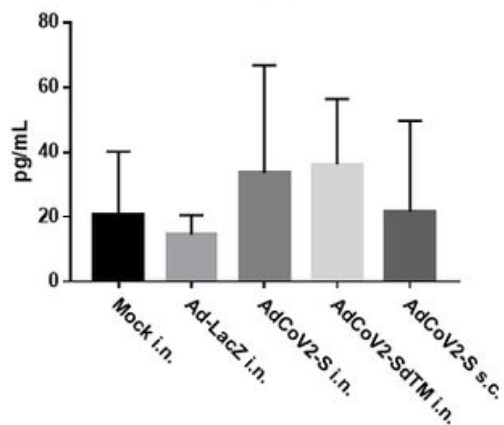

IL-4

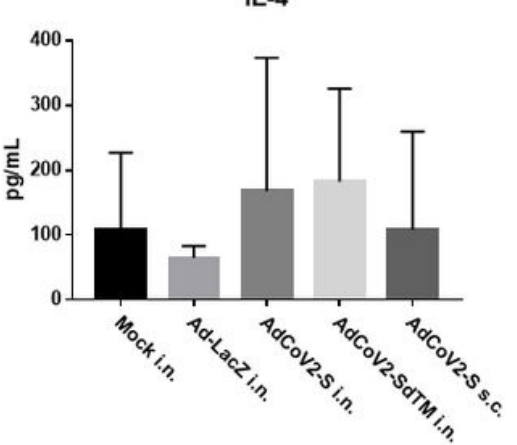

Figure 8

"See the Supplemental Files section for the complete figure caption".

\section{Supplementary Files}

This is a list of supplementary files associated with this preprint. Click to download.

- Supplementaryinformation.pdf

- FigureCaptions.pdf 